\title{
Cellular uptake and fate of fibroin microspheres loaded with randomly fragmented DNA in 3T3 cells
}

This article was published in the following Dove Press journal:

International Journal of Nanomedicine

13 May 2016

Number of times this article has been viewed

Jin Sil Lee

Won Hur

Department of Bioengineering and Technology, Kangwon National University, Chuncheon, South Korea
Correspondence: Won Hur Department of Bioengineering and Technology, Kangwon National University, Chuncheon 200-70I, South Korea

Tel +82 332506276

Fax +82332436350

Email wonhur@kangwon.ac.kr
Abstract: Purified fibroin protein can be obtained in large quantities from silk fibers and processed to form microscopic particles as delivery vehicles for therapeutic agents. In this study, we demonstrated that fibroin microspheres were taken up by $3 \mathrm{~T} 3$ cells, localized in the nonlysosomal compartment, and secreted from the cytoplasm after medium replenishment. DNA-loaded microspheres were taken up by $>95 \%$ of 3 T3 cells. DNA cargo had no influence on the intracellular trafficking of microspheres, while fluorescently labeled cargo DNA was observed in the lysosomal compartment and in the microspheres. These results indicate that fibroin microspheres can travel through $3 \mathrm{~T} 3$ cells without making any contact with the lysosomal compartments. The amount of DNA loaded in the microspheres taken up by 3T3 cells was estimated up to $831.0 \mathrm{pg} / \mathrm{cell}$. Thus, fibroin microspheres can deliver a large amount of randomly fragmented DNA $(<10 \mathrm{~kb})$ into the cytoplasmic compartment of 3T3 cells.

Keywords: microspheres, DNA delivery, transcellular transport, fibroin

\section{Introduction}

Cells take up microspheres made of natural ${ }^{1}$ or synthetic polymers. ${ }^{2,3}$ Thus, microspheres have been developed as vehicles not only for gene transfection ${ }^{4}$ but also for small interfering RNAs (siRNAs) ${ }^{5}$ and decoy DNA. ${ }^{6}$ However, gene transfection and siRNA delivery largely rely on nanoparticles, for example, a biodegradable arginine-based polymer showing an enhanced transfection efficiency ${ }^{7}$ and a cationic core and lipid shell providing a long-circulating nanoparticle platform. ${ }^{8}$ Although nanoparticles are considered to be better candidates for nonviral gene delivery because of their enhanced cellular internalization and endosomal escape, ${ }^{5}$ micron-scale spheres may have advantages in terms of delivering large and multiple cargoes, including not only genetic materials but also mixtures of macromolecules that may modulate cellular metabolism.

Endocytosed polymeric molecules are subject to lysosomal degradation unless endosomal escape occurs, ${ }^{9}$ whereas the fate of microspherical vehicles varies, depending on the size and host cells. Microparticles phagocytosized by macrophages were trafficked to phagolysosomes. ${ }^{10}$ Disulfide-stabilized poly(methacrylic acid) capsules underwent lysosomal degradation by colon cancer cells. ${ }^{11}$ However, latex particles of $500 \mathrm{~nm}$ in diameter were not localized in the lysosomal compartment in murine melanoma cell line B16-F10. ${ }^{12}$ Thus, understanding the intracellular trafficking and fate of microspheres is important to design delivery system.

In a previous report, fibroin microspheres were fabricated without any chemical modification or cross-linking in order not to disturb the proteinous surface of the 
fibroin shell. ${ }^{13}$ These microspheres are efficiently taken up by $3 \mathrm{~T} 3$ cells with no significant loss of cell viability. ${ }^{1}$ However, little is known about the intracellular fate and DNA delivery capability of fibroin microspheres. In this study, we investigated the cellular uptake and fate of fibroin microspheres in $3 \mathrm{~T} 3$ cells. We also investigated the subcellular localization of fibroin microspheres loaded with and without randomly fragmented DNA.

\section{Materials and methods Fibroin microspheres}

Aqueous fibroin solution was prepared from silk fibers using hot $\mathrm{CaCl}_{2}$ solutions as reported previously. ${ }^{13}$ Fluorescein isothiocyanate (FITC)-dextran (40 kDa), Span 80, Tween 80, and other reagents were purchased from Sigma-Aldrich Co. (St Louis, MO, USA). Salmon testis chromosomal DNA (D1626 41.2\% GC; Sigma-Aldrich Co.) was used to prepare randomly fragmented DNA using a probe-type sonicator set at 500 W (VC505; Sonics \& Materials Inc., Newtown, CT, USA) for 0-60 seconds in pulse mode and at an amplitude of $40 \%$. FITC-labeled DNA was prepared by sonication of DNA fragments $\left(20 \mathrm{mg}\right.$ ) solubilized in $4 \mathrm{~mL}$ of $\mathrm{NaHCO}_{3}$ buffer $(0.1 \mathrm{M}, \mathrm{pH} 9)$ for 60 seconds and reacted with $120 \mu \mathrm{L}$ of FITC ( $1 \mathrm{mg} / \mathrm{mL}$ prepared in dimethyl sulfoxide) for 1 hour at $4^{\circ} \mathrm{C}$ in the dark, followed by Sephadex G25 chromatography to separate unreacted FITC.

Microspheres were prepared from aqueous fibroin solution $(2 \% \mathrm{w} / \mathrm{v})$ or that supplemented with fragmented DNA, FITC-labeled DNA, or FITC-dextran. An $8 \mathrm{~mL}$ of fibroin solution was mixed with an organic phase prepared by dissolving a surfactant mixture of $2.2 \mathrm{~mL}$ of Span 80 and $1.8 \mathrm{~mL}$ of Tween 80 in $16 \mathrm{~mL}$ of $n$-decane (decane) at $40^{\circ} \mathrm{C}$, followed by emulsification of the oil phase using a handheld homogenizer (Polytron PT2100; Kinematica, Lucerne, Switzerland) at 30,000 rpm for 60 seconds. The resulting translucent emulsion was dried using a rotary vacuum evaporator (JP/N 1000S-W; Eyela, Tokyo, Japan) at $40^{\circ} \mathrm{C}$ for 30 minutes. The microspheres that formed in the emulsion were harvested by centrifugation at 4,000 rpm for 5 minutes, washed several times with ethanol to remove decane, and dried at room temperature (Figure S1). The microspheres were characterized using scanning electron microscopy (S-4800; Hitachi Ltd, Tokyo, Japan) and transmission electron microscopy (JEM-2100F; JEOL, Tokyo, Japan). The zeta potential of fibroin microspheres was measured using an electrophoretic light-scattering spectrophotometer (ELS-8000; Otsuka, Tokyo, Japan). Electrophoresis was carried out in 1\% agarose gel. Microspheres were mixed with loading buffer
( $0.3 \%$ bromophenol blue and 20\% glycerol) and directly loaded into the wells of a precast gel.

\section{T3 cell culture and microsphere uptake}

The BALB/3T3 clone A31 cell line was purchased from American Type Culture Collection (Manassas, VA, USA) and maintained in Dulbecco's Modified Eagle's Medium containing $4.5 \mathrm{~g} / \mathrm{L}$ glucose and $4 \mathrm{mM}$ L-glutamine (Lonza, Walkedrsville, MD, USA) and supplemented with $10 \%$ fetal bovine serum (Lonza) at $37^{\circ} \mathrm{C}$ in a humidified atmosphere consisting of $5 \% \mathrm{CO}_{2}$ in air. Microspheres were added at a final concentration of $0.33 \mathrm{mg} / \mathrm{mL}$ (unless otherwise described) to a monolayer of 3T3 cells grown for 24 hours in six-well culture plates seeded with $10^{5}$ cells per well or in a $60 \mathrm{~mm}$ culture dish seeded with $10^{6}$ cells. The cells were further incubated for a given amount of time and then washed twice with phosphate-buffered saline (PBS). Cells were detached by trypsin-ethylenediaminetetraacetic acid treatment for flow cytometric analysis.

\section{Fluorescence microscopy}

Cells were cultured with microspheres in a 12-well culture plate containing coverslips. The coverslips were recovered from the culture wells and incubated with a fixing solution (4\% formaldehyde). Fluorescence images of the samples were obtained using confocal laser scanning microscopy (CLSM; FluoView FV300; Olympus Corporation, Tokyo, Japan). For lysosome staining, cells were replenished with medium (1 mL) containing LysoTracker Red DND-99 (50 nM; Molecular Probes Inc., Eugene, OR, USA) and further incubated for 30 minutes at room temperature. Cells were washed with PBS and fixed with formaldehyde prepared in PBS for 30 minutes at room temperature.

\section{Flow cytometric analysis}

Trypsinized cell suspensions were washed with PBS to remove extracellular microspheres and cell debris and then fixed in 4\% formaldehyde. Cells were washed with cold PBS and resuspended in PBS for flow cytometric analysis using a FACSCalibur flow cytometer (BD Biosciences, San Jose, CA, USA). Matlab software (Mathworks, Natick, MA, USA) was used to analyze raw data and to generate scatter dot plots of forward scatter, side scatter, and FL1 signals. The percentage of cells with internalized microspheres was calculated by the number of fluorescence events of FL1height signals above a threshold level of background fluorescence. A specified volume of spent culture medium was also used for fluorescence-activated cell sorting to monitor 
extracellular secretion of microspheres or fluorescent cargoes. The results were expressed as the mean \pm standard error. Statistical difference in the fluorescence intensity was determined using one-way analysis of variance with Dunnett's post hoc test. Differences were considered statistically significant at $P<0.05$.

\section{Results}

\section{Preparation and cellular uptake of fibroin microspheres}

Regenerated fibroin was mixed with FITC-dextran (2.5\%) to prepare core-shell morphology microspheres. The resulting microspheres were smooth and spherical with number- and weight-averaged diameters of $0.73 \mu \mathrm{m}$ and $1.63 \mu \mathrm{m}$, respectively (Figure 1). The zeta potential was determined to be $6.09 \pm 1.44 \mathrm{mV}$. CLSM showed that the microspheres had fluorescent cores, indicating the formation of a core-shell structure with FITC-dextran core surrounded by fibroin, consistent with a previous report. ${ }^{13}$ The microspheres were added to the culture medium in which $3 \mathrm{~T} 3$ cells were cultured for 24 hours. During subsequent incubation, microspherical bodies being localized in the cytoplasm of cells were observed using an inverted phase-contrast microscope (Video S1). Confocal microscopy confirmed that the cytoplasm contained fluorescent foci, the sizes of which were comparable to those of fibroin microspheres, indicating the uptake of multiple FITC-dextran-containing microspheres (Figure 2). Transmission electron microscopy also confirmed the cytoplasmic localization of these microspheres in $3 \mathrm{~T} 3$ cells (Figure 2B). The microspheres remained in the cytoplasm for up to 7 days, unless the culture medium was not replenished.

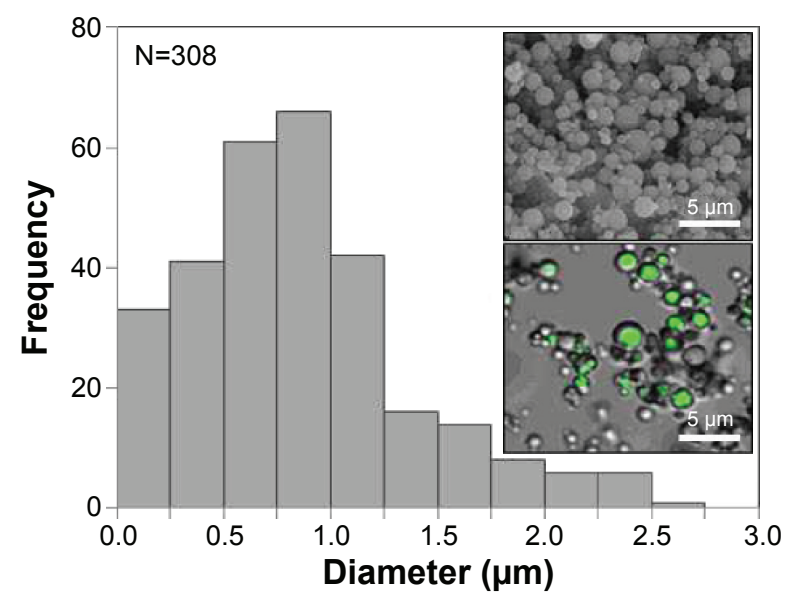

Figure I Size histogram of fibroin microspheres and scanning electron and confocal micrographs (inserts).
As soon as the culture medium was replenished, disappearance of the spherical bodies in the cytoplasm was observed by inverted microscopy (Figure 3A, Figure S2, and Video S2). Flow cytometry was used to quantify cells that had taken up fluorescent microspheres and to trace FITC fluorescence (Figure 3B). Cells treated with FITC-dextrancontaining microspheres $(0.1 \mathrm{mg} / \mathrm{mL})$ were incubated for 24 hours, after which the medium was replenished. The relative fluorescence intensity of the replenished culture medium increased with time and reached a plateau after 2 hours (Figure 3C). The relative fluorescence intensity of the spent culture medium corresponded to up to $\sim 15 \%$ of that of the cells. Flow cytometry also showed that most cells remained fluorescent after medium replenishment. Thus, cells were treated with a low dose of microspheres $(0.01 \mathrm{mg} / \mathrm{mL})$, incubated with daily medium replenishment, and subjected to flow cytometric analysis. The percentage of fluorescent cells was $66.0 \% \pm 4.1 \%, 22.5 \% \pm 5.9 \%$, $13.5 \% \pm 3.0 \%$, and $6.9 \% \pm 1.6 \%$ after the medium was replenished one, two, three, and four times, respectively (Figure 3D). The spent culture medium was also analyzed by flow cytometry to detect fluorescence. The fluorescence intensity of the spent culture medium was gated based on the microsphere size to determine the fluorescence intensity of microspheres secreted by cells. The fluorescence intensity of the first, second, third, and fourth spent culture medium to be collected was corresponded to $21.0 \% \pm 6.7 \%$, $4.32 \% \pm 1.8 \%, 0.51 \% \pm 0.10 \%$, and $0.88 \% \pm 0.3 \%$, respectively, of the fluorescence intensity of microspheres added to the culture medium. Accordingly, the cumulative fluorescence intensity increased with each change and reached a plateau of $26.7 \%$.

Qualitative and quantitative estimation of microspheres that were taken up and secreted by cells were determined by microscopic observations and flow cytometry. These results clearly indicate that fibroin microspheres are taken up by $3 \mathrm{~T} 3$ cells and secreted upon medium replenishment. The microspheres are retained in the cytoplasm as far as the culture medium contains microspheres at a high concentration. Accordingly, it suggests a possibility that ingested microspheres are in dynamic equilibrium with those in the culture medium.

\section{Effect of DNA cargo on cellular uptake and secretion of microspheres}

Fibroin microspheres were prepared to contain a mixture of randomly fragmented salmon testis DNA. The fibroin microspheres loaded with DNA fragments were stained 

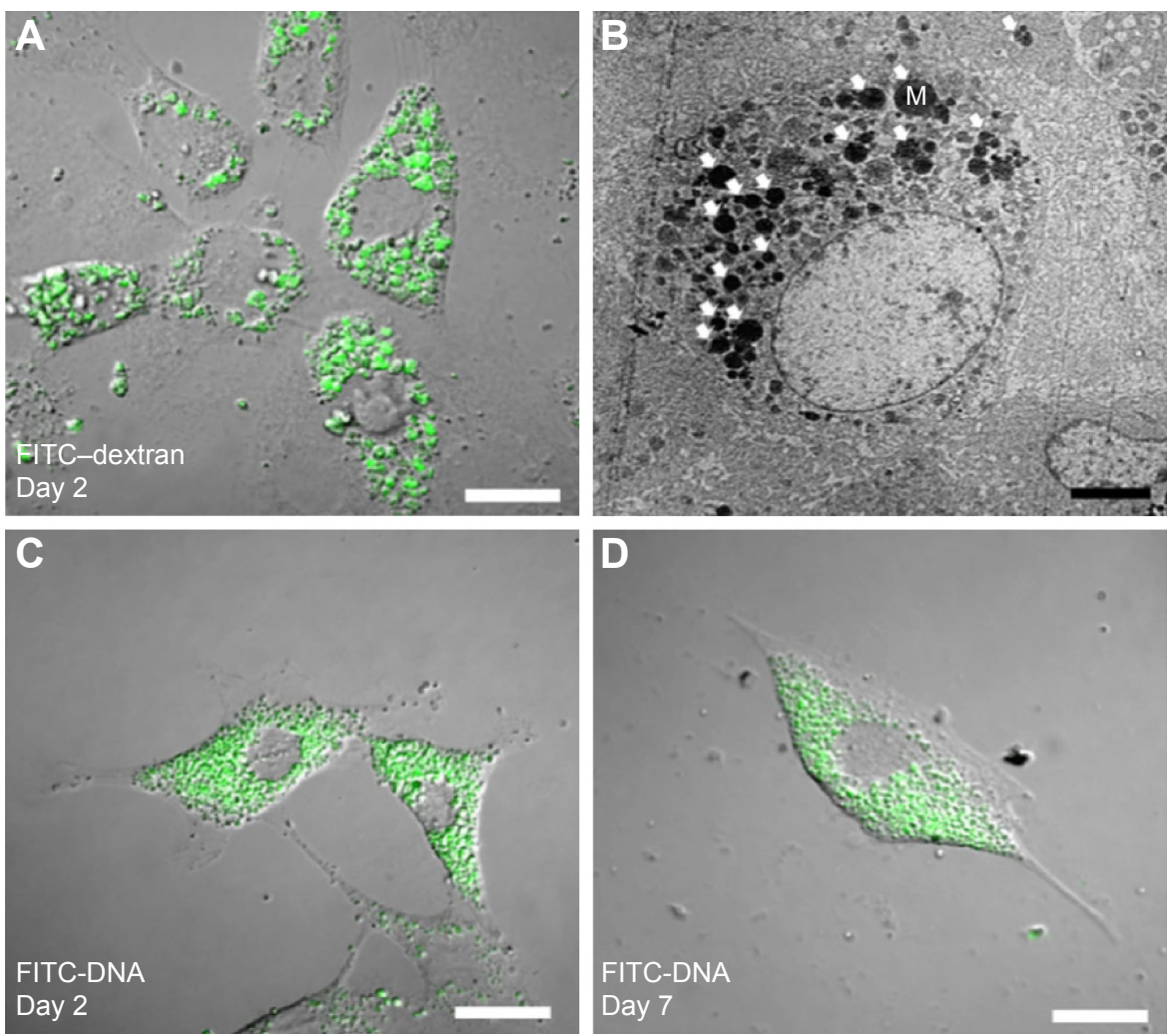

Figure 2 Microspheres localized in the cytoplasm of 3T3 cells.

Notes: Differential interference contrast and fluorescence overlay image (A) and transmission electron micrograph (B) of 3T3 cells treated with FITC-dextran-containing microspheres (white arrows). Overlay images of 3 T3 cells treated with FITC-DNA-containing microspheres at day 2 (C) and day 7 (D). Scale bars are $20 \mu \mathrm{m}$ in (A), (C), and (D), and $5 \mu \mathrm{m}$ in (B).

Abbreviations: FITC, fluorescein isothiocyanate; $\mathrm{M}$, microspheres.

with FITC and ethidium bromide. CLSM revealed that the DNA fragments were evenly distributed throughout the microspheres (Figure 4A). The size distributions of DNA fragments eluted from microspheres were significantly different from those of DNA fragments that were used to prepare microspheres, indicating that DNA fragments $>10 \mathrm{~kb}$ could not be released from the microspheres by electrophoresis (Figure 4B). Densitometry analysis using image J software indicated that the percentage of DNA released by electrophoresis was $52.6 \%$.

The effect of cargo DNA on the cellular uptake and secretion of fibroin microspheres was investigated using

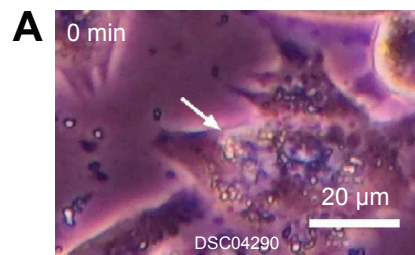

B

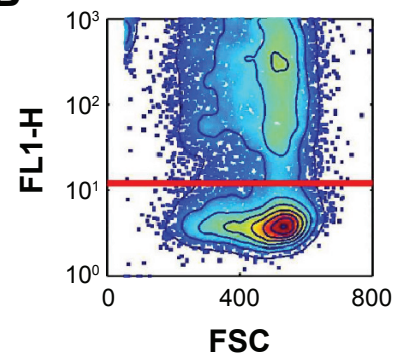

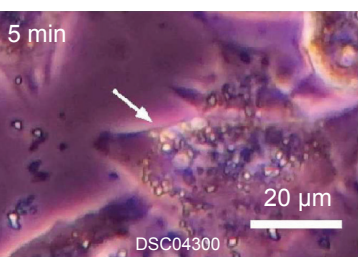

Medium

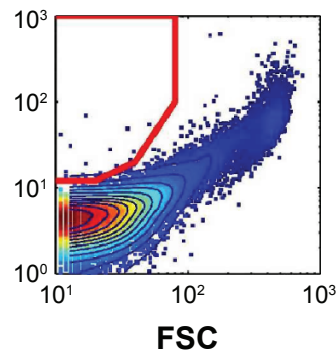

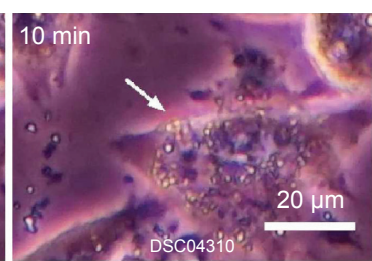

Microsphere

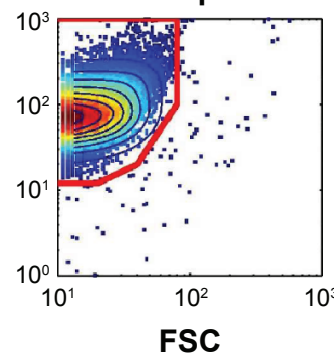

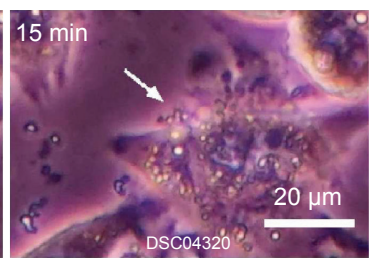

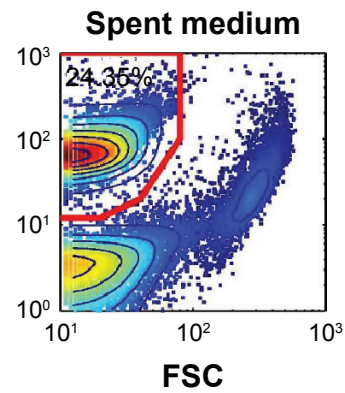

Figure 3 (Continued) 
C

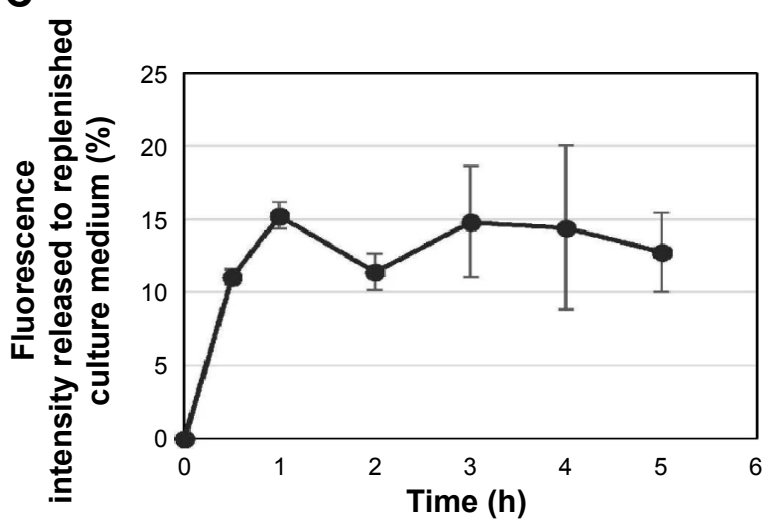

D

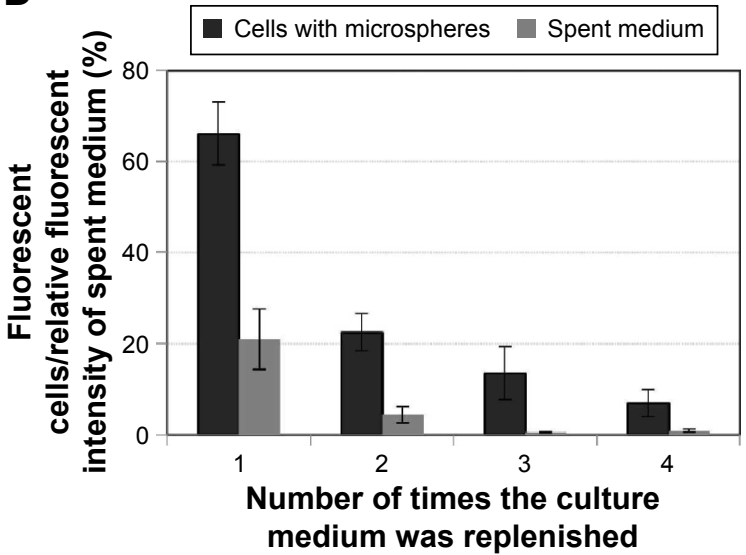

Figure 3 Cellular uptake and release of fibroin microspheres.

Notes: A series of inverted phase-contrast microscope images of 3T3 cells secreting microspheres after medium replenishment (A). Arrows indicate secretion of microspheres. Scatter plots of FLI-height (FLI-H; FITC fluorescence) versus forward scatter (FSC) of cells, culture media, microspheres, and spent culture media (B). A time course of the relative fluorescence intensity of the replenished culture medium of cells treated with FITC-dextran-containing microspheres (C). The percentage of fluorescent cells and the fluorescence intensity of the spent culture medium according to the number of times the medium was replenished (D).

Abbreviations: FITC, fluorescein isothiocyanate; FSC, forward scatter; min, minutes; h, hours.

FITC-dextran-containing microspheres loaded with different amounts of fragmented DNA. Since fibroin and DNA molecules were dissolved in aqueous droplets in water-in-oil emulsion in the process of microsphere preparation, DNA molecules are quantitatively retained in the microspheres.
These were added to $3 \mathrm{~T} 3$ cells, the cells were incubated for 24 hours, and then the medium was replenished. After 24 hours, the cells and spent culture medium were harvested and analyzed by flow cytometry. Most cells were fluorescent indicating uptake of fluorescent microspheres (Figure 4C).
A
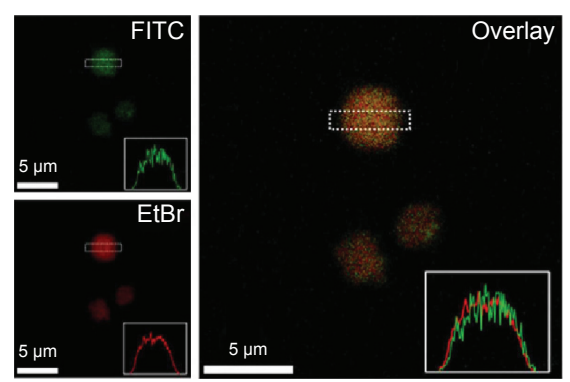

C

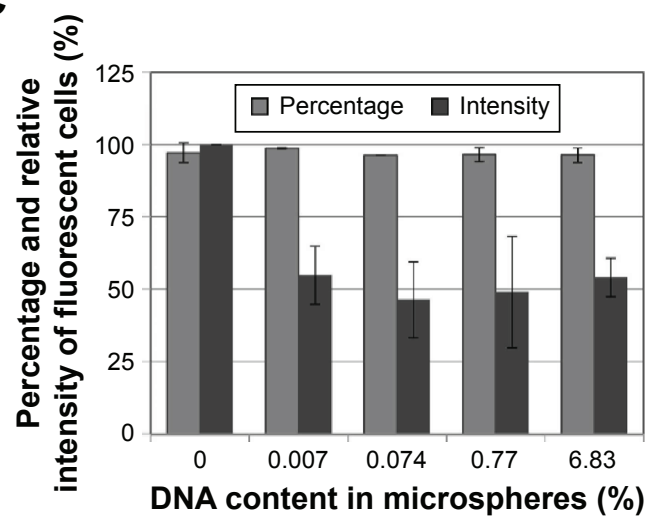

B

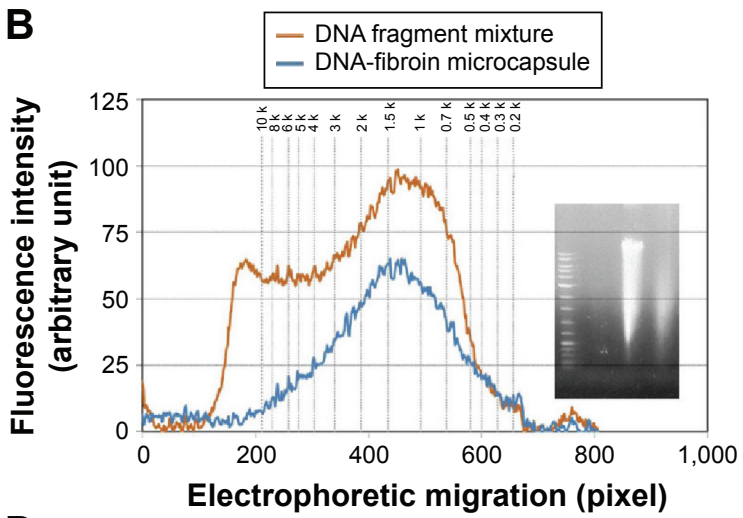

D

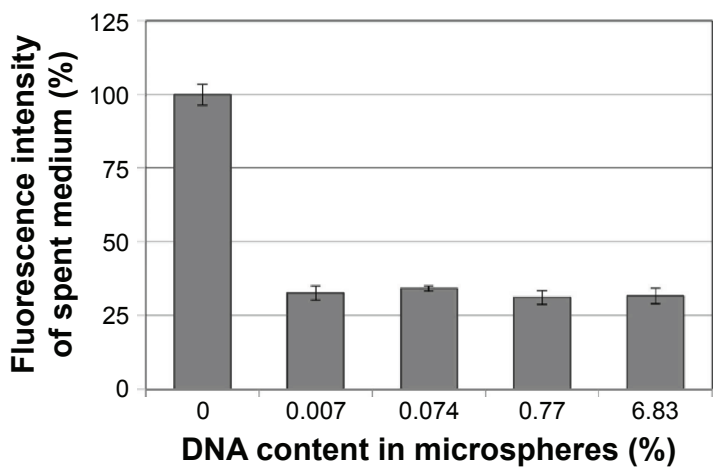

Figure 4 Preparation and cellular uptake of DNA-containing microspheres.

Notes: Confocal microscopy images of DNA-containing fibroin microspheres stained with EtBr and labeled with FITC (A). Size distribution of DNA fragments used to prepare microspheres and released DNA fragments using electrophoresis (B). Percentage and fluorescence intensity of fluorescent cells following treatment with FITC-dextran-containing microspheres containing different amounts of fragmented DNA (C). Fluorescence intensity of the replenished culture medium after 24 hours (D). Abbreviations: $\mathrm{EtBr}$, ethidium bromide; FITC, fluorescein isothiocyanate. 
However, cellular fluorescence intensity was decreased to $55.4 \% \pm 19.5 \%, 47.9 \% \pm 22.6 \%, 36.3 \% \pm 9.4 \%$, and $53.1 \% \pm 6.2 \%$ in the cells treated with microspheres loaded with $0.007 \%, 0.074 \%, 0.71 \%$, and $6.82 \%$ fragmented DNA, respectively. Flow cytometry also estimated the amount of DNA loaded in the microspheres taken up by cells, which were $0.27 \mathrm{pg} / \mathrm{cell}, 5.1 \mathrm{pg} / \mathrm{cell}, 66.3 \mathrm{pg} / \mathrm{cell}$, and $831.0 \mathrm{pg} /$ cell. DNA loading also decreased the fluorescence intensity of the spent culture medium by approximately threefold in comparison to that of the control (Figure 4D). These results demonstrate that cellular uptake and extracellular secretion of microspheres were inhibited by the presence of DNA molecules in microspheres.

We further investigated the effect of DNA fragment size on the uptake and secretion of microspheres. FITCdextran-containing microspheres were fabricated to contain fragmented DNA that was prepared by differential sonication (Figure 5A). More than $90 \%$ of DNA was fragmented to shorter than $10 \mathrm{~kb}$ and $3 \mathrm{~kb}$ by sonication for 30 seconds and 60 seconds, respectively. FITC-dextrancontaining microspheres containing this fragmented DNA $(6.8 \%)$ were prepared and added to 3 T3 cells. Flow cytometry determined that most cells became fluorescent (Figure 5B); however, fluorescence intensity was significantly higher in cells treated with microspheres containing shorter DNA fragments (sonicated for 60 seconds) than in cells treated with microspheres containing longer DNA fragments (sonicated for 30 seconds) (Figure 5C). The fluorescence intensity of the spent culture media was decreased from $11.76 \% \pm 1.44 \%$ to $9.15 \% \pm 1.93 \%$ and $8.30 \% \pm 0.77 \%$ following the treatment of cells with microspheres containing DNA that had been sonicated for 30 seconds and 60 seconds, respectively (Figure 5D). Accordingly, shorter DNA fragments facilitated the cellular uptake of microspheres and inhibited the extracellular removal of microspheres themselves and/or released

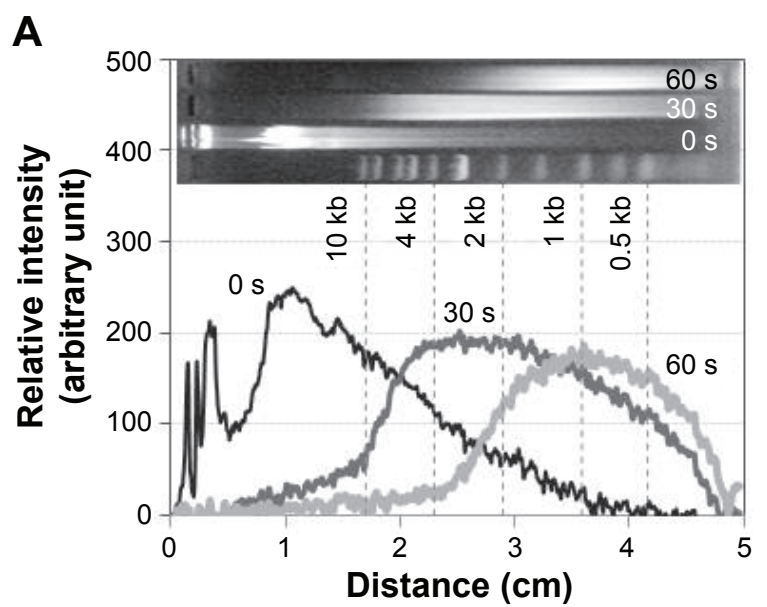

B
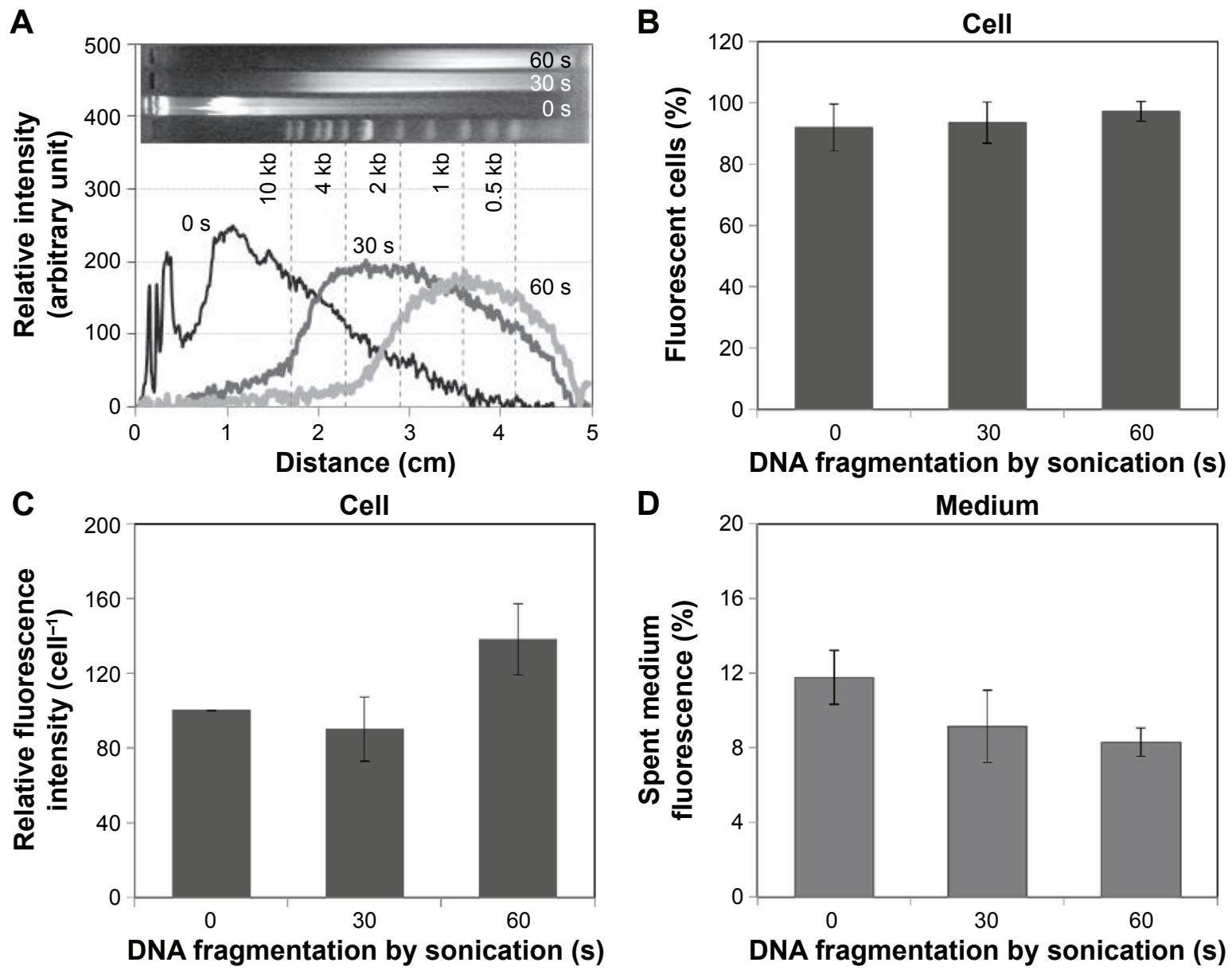

Figure 5 The effect of cargo DNA fragmentation on microsphere ingestion.

Notes: Densitometry of DNA fragments prepared by sonication for $0 \mathrm{~s}, 30 \mathrm{~s}$, and $60 \mathrm{~s}$ (A). Percentage (B) and relative fluorescence intensity (C) of fluorescent cells following treatment with FITC-dextran-containing microspheres containing fragmented DNA prepared by sonication for $0 \mathrm{~s}$, $30 \mathrm{~s}$, and $60 \mathrm{~s}$. Fluorescence intensity of the replenished media after 24 hours (D).

Abbreviations: FITC, fluorescein isothiocyanate; s, seconds. 


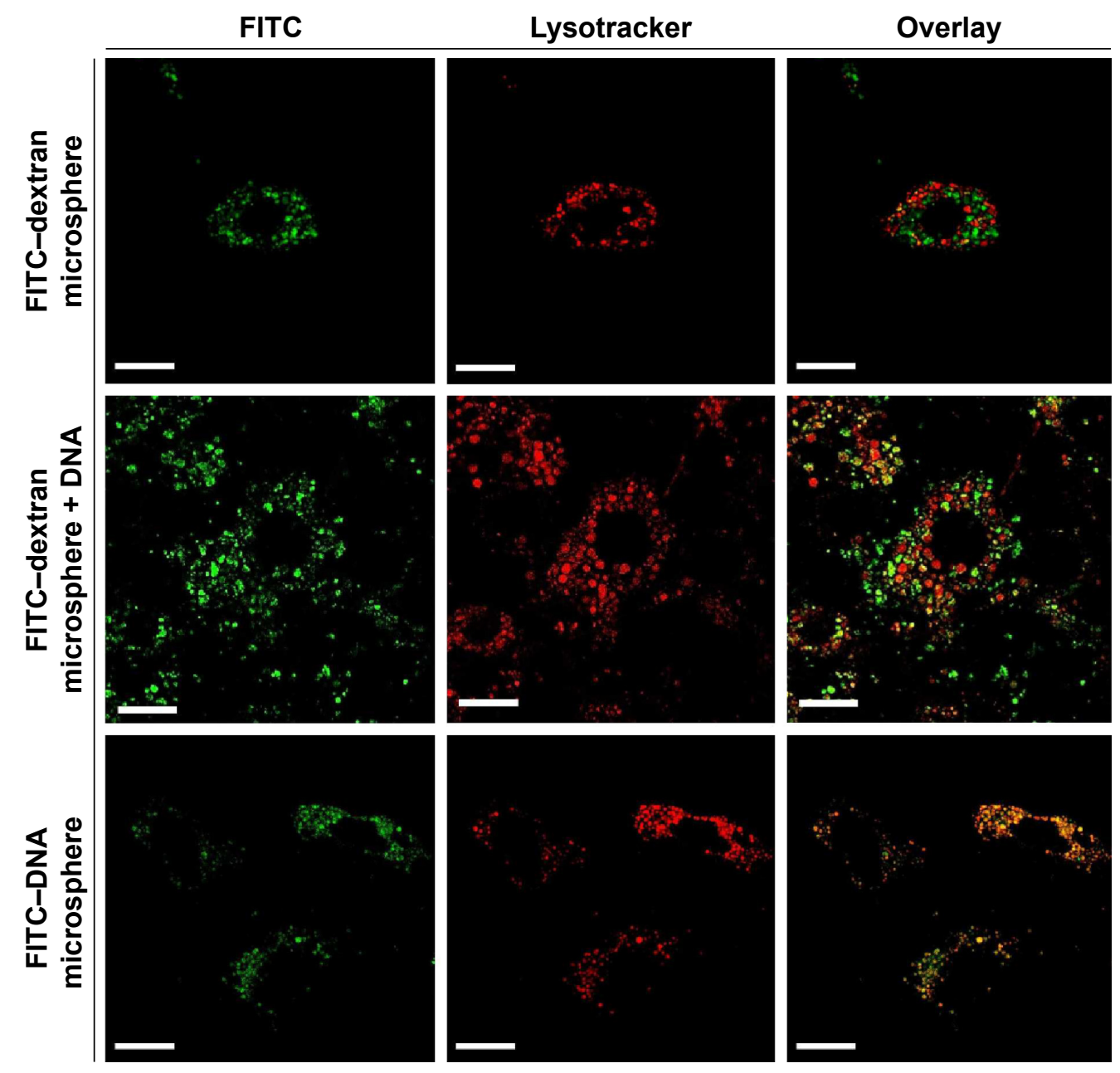

Figure 6 Fluorescence and overlay images of 3 T3 cells stained with LysoTracker Red following treatment with FITC-dextran-containing microspheres without DNA (top row), FITC-dextran-containing microspheres with DNA (middle row), and FITC-DNA-containing microspheres (bottom row).

Note: Magnification $\times 1000$; scale bar $=10 \mu \mathrm{m}$.

Abbreviation: FITC, fluorescein isothiocyanate.

FITC-dextran. These results show that the fluorescence of the spent medium is not always proportional to the number of microspheres taken up by the cells.

\section{The effect of DNA cargo on the subcellular localization of microspheres}

Cells treated with microspheres $(0.1 \mathrm{mg} / \mathrm{mL})$ were harvested and stained with LysoTracker Red, a pH-sensitive dye that emits red fluorescence in lysosomes, to trace the subcellular localization of microspheres. CLSM images in the respective wavelength channels (FITC and LysoTracker Red) and the overlay of these images on to representative cell images are shown in Figure 6. The overlay image of 3T3 cells treated with FITC-dextran-containing microspheres with and without DNA shows green and red fluorescent foci in cytoplasmic compartments (top and middle row of Figure 6). This result indicates that fibroin microspheres were localized in the nonlysosomal compartment. Thus, DNA cargo does not seem to affect the subcellular localization of fibroin microspheres. Fibroin microspheres containing only FITC-labeled fragmented DNA were prepared and added to cells. CLSM showed that the cells contained orange-colored foci and green foci (bottom row of Figure 6), indicating the lysosomal localization of FITC-DNA. This suggests that a portion of FITC-DNA was released from microspheres and transported to the endocytic lysosomal compartment. Accordingly, these results demonstrate that, following cellular uptake, fibroin microspheres were not routed to the lysosomal compartment.

\section{Discussion}

Fibroin microspheres were taken up and resided in the nonlysosomal compartment of $3 \mathrm{~T} 3$ cells. The observation is similar to large latex particles $(500 \mathrm{~nm})$ that were internalized by caveolae-mediated endocytosis and were not directed to the lysosomal compartment in nonphagocytic cells. ${ }^{12}$ The authors 
also showed that small latex particles $(<200 \mathrm{~nm})$ were subjected to lysosomal degradation. Some bacterial pathogens enter cells via caveolae-mediated endocytosis, thereby escaping degradation in the lysosomal compartment. ${ }^{14}$ Similarly, in this study, internalized fibroin microspheres were not routed to lysosomes. However, internalized nanoparticles mostly proceed through the endocytic pathway toward lysosomal compartments. Accordingly, size seems to be a critical factor that determines the endocytic pathway. Further investigations are needed to confirm that the entry pathway of fibroin microspheres underlies the delivery of these particles to the cytoplasm, not to lysosomes.

Cellular secretion of micron-sized particles has been seldom investigated, whereas nanoparticle exocytosis has been extensively studied. Internalized nanoparticles can be actively transported to the periphery of cells and exocytosed or fused with lysosomes and other cytoplasmic compartments that have a slow turnover. ${ }^{15,16}$ The localization of nanoparticles into organelles is a key factor in exocytosis. ${ }^{17}$ In this study, fibroin microspheres were secreted into the culture media upon medium replenishment and were eventually removed by repeated medium changes. Taken together, microsphere secretion may have a different trafficking pathway to secretion.

Fragmented DNA molecules were distributed evenly throughout microspheres, and $>50 \%$ was released by electrophoresis. Thus, DNA cargo can be released to cytoplasm and subjected to lysosomal degradation. Exogenous doublestranded DNA is recognized by Toll-like receptor 9 and is transported to lysosomes for degradation. ${ }^{18,19}$ This is similar to the innate immune responses that eliminate viruses and other intracellular microbes via endolysosomal trafficking and degradation. ${ }^{20}$ Thus, we can expect that DNA fragments released from microspheres have certain effects on cellular uptake and secretion of microspheres.

This study also showed that the size of cargo DNA influenced the cellular uptake and secretion of microspheres; small DNA fragments facilitated microsphere uptake and inhibited their extracellular secretion by 3T3 cells. Microspheres contained an identical amount of differentially fragmented salmon testis DNA; therefore, the number of small DNA fragments was significantly higher than that of less fragmented DNA. Diffusion of large DNA fragments is much slower than that of smaller DNA fragments in the crowded cytosol with actin networks. ${ }^{21}$ Thus, a large amount of small DNA fragments easily released from microspheres might be responsible for the effects of cargo DNA size on microsphere uptake and secretion; however, the detailed mechanism was not elucidated.

\section{Conclusion}

This study demonstrated that fibroin microspheres were taken up by $3 \mathrm{~T} 3$ cells, retained in the nonlysosomal cytoplasmic compartment, and secreted into the culture medium. The intracellular trafficking was not influenced by the presence of cargo DNA in the microspheres. Cargo DNA decreased the amounts of microspheres taken up and secreted by $3 \mathrm{~T} 3$ cells but had no effect on the percentage of cells that ingested microspheres.

Accordingly, fibroin microspheres have the potential to be developed as a means to modulate and monitor intracellular physiology. Because of their micron-scale size, fibroin microspheres can be developed as an intracellular delivery vehicle for complicated cargoes such as mixtures of nucleases and DNA that continuously release decoy molecules, siRNA or microRNA sponges for gene expression control, and unmethylated CpG DNA for activating immune cells. Since fibroin microspheres travel through cells without undergoing lysosomal degradation, secreted microspheres may contain some cytoplasmic molecules that enable us to monitor intracellular physiology.

\section{Acknowledgment}

This research was supported by the Basic Science Research Program through the National Research Foundation of Korea (NRF) funded by the Ministry of Education, Science, and Technology (NRF-2013R1A1A2074373).

\section{Disclosure}

The authors report no conflicts of interest in this work.

\section{References}

1. Go NK, Lee LS, Lee JH, Hur W. Growth, cell cycle progression, and morphology of $3 \mathrm{~T} 3$ cells following fibroin microsphere ingestion. J Biomed Mater Res A. 2015;103(4):1325-1331.

2. Alexander LM, Pernagallo S, Livigni A, Sánchez-Martín RM, Brickman JM, Bradley M. Investigation of microsphere-mediated cellular delivery by chemical, microscopic and gene expression analysis. $\mathrm{Mol}$ Biosyst. 2010;6(2):399-409.

3. Palamà IE, Coluccia AML, della Torre A, et al. Multilayered polyelectrolyte capsules and coated colloids: cytotoxicity and uptake by cancer cells. Sci Adv Mater. 2010;2:138-150.

4. Putnam D. Polymers for gene delivery across length scales. Nat Mater. 2006;5(6):439-451.

5. Présumey J, Salzano G, Courties G, et al. PLGA microspheres encapsulating siRNA anti-TNFalpha: efficient RNAi-mediated treatment of arthritic joints. Eur J Pharm Biopharm. 2012;82(3):457-464.

6. De Stefano D, De Rosa G, Maiuri MC, et al. Oligonucleotide decoy to NF- $\kappa \mathrm{B}$ slowly released from PLGA microspheres reduces chronic inflammation in rat. Pharmacol Res. 2009;60(1):33-40.

7. Wu J, Yamanouchi D, Liu B, Chu CC. Biodegradable arginine-based poly (ether ester amide)s as a non-viral DNA delivery vector and their structure-function study. J Mater Chem. 2012;22:18983-18991.

8. Zhu X, Xu Y, Solis LM, et al. Long-circulating siRNA nanoparticles for validating Prohibitin1-targeted non-small cell lung cancer treatment. Proc Natl Acad Sci U S A. 2015;112(25):7779-7784. 
9. Varkouhi AK, Scholte M, Storm G, Haisma HJ. Endosomal escape pathways for delivery of biological. J Control Release. 2011;151(3): 220-228.

10. Jay SM, Skokos EA, Zeng J, Knox K, Kyriakides TR. Macrophage fusion leading to foreign body giant cell formation persists under phagocytic stimulation by microspheres in vitro and in vivo in mouse models. J Biomed Mater Res A. 2010;93(1):189-199.

11. Yan Y, Johnston AP, Dodds SJ, et al. Uptake and intracellular fate of disulfide-bonded polymer hydrogel capsules for doxorubicin delivery to colorectal cancer cells. ACS Nano. 2010;4(5):2928-2936.

12. Rejman J, Oberle V, Zuhorn IS, Hoekstra D. Size-dependent internalization of particles via the pathways of clathrin- and caveolae-mediated endocytosis. Biochem J. 2004;377(Pt 1):159-169.

13. Kim EJ, Lee SY, Hur W. Preparation of core-shell microcapsules using nanodispersed fibroin. J Appl Polym Sci. 2011;121:3460-3465.

14. Shin JS, Abraham SN. Caveolae as portals of entry for microbes. Microbes Infect. 2001;3(9):755-761.

15. Panyam J, Labhasetwar V. Dynamics of endocytosis and exocytosis of poly (D, L-lactide-co-glycolide) nanoparticles in vascular smooth muscle cells. Pharm Res. 2003;20(2):212-220.
16. Cartiera MS, Johnson KM, Rajendran V, Caplan MJ, Saltzman WM. The uptake and intracellular fate of PLGA nanoparticles in epithelial cells. Biomaterials. 2009;30(14):2790-2798.

17. Sakhtianchi R, Minchin RF, Lee KB, Alkilany AM, Serpooshan V, Mahmoudi M. Exocytosis of nanoparticles from cells: role in cellular retention and toxicity. Adv Colloid Interface Sci. 2013;201:18-29.

18. Krug A, Luker GD, Barchet W, Leib DA, Akira S, Colonna M. Herpes simplex virus type 1 activates murine natural interferon-producing cells through toll-like receptor 9. Blood. 2004;103(4):1433-1437.

19. Lund J, Sato A, Akira S, Medzhitov R, Iwasaki A. Toll-like receptor 9-mediated recognition of Herpes simplex virus-2 by plasmacytoid dendritic cells. J Exp Med. 2003;198(3):513-520.

20. Kawai T, Akira S. Innate immune recognition of viral infection. Nat Immunol. 2006;7(2):131-137.

21. Dauty E, Verkman AS. Actin cytoskeleton as the principal determinant of size-dependent DNA mobility in cytoplasm. J Biol Chem. 2005; 280(9):7823-7828. 


\section{Supplementary materials}

Video SI The movie shows inverted phase-contrast time-lapse microscopic images of 3T3 cells in a six-well culture plate fed with fibroin microspheres. Arrows indicate cellular uptake of microspheres. The time-lapse covers a period of 29.7 minutes (AVI $400 \times 400 ; 4.6 I$ MB).

Video S2 The movie shows inverted phase-contrast time-lapse microscopic images of microsphere-ingested 3T3 cells in a six-well culture plate after medium replenishment. Arrows indicate cellular secretion of microspheres. The time-lapse covers a period of I 72 minutes (AVI 400×400; 3.97 MB)

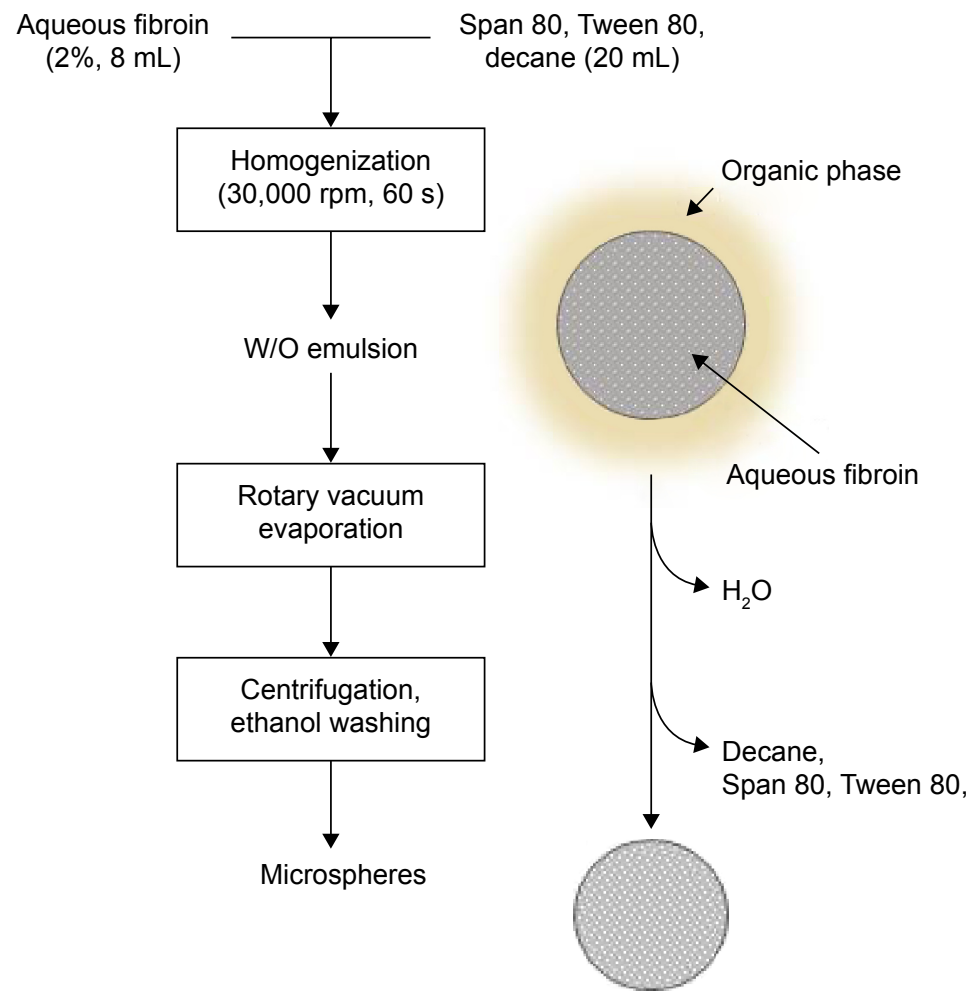

Figure SI A schematic procedure for the preparation of fibroin microspheres.

Abbreviations: s, seconds; W/O, water-in-oil. 

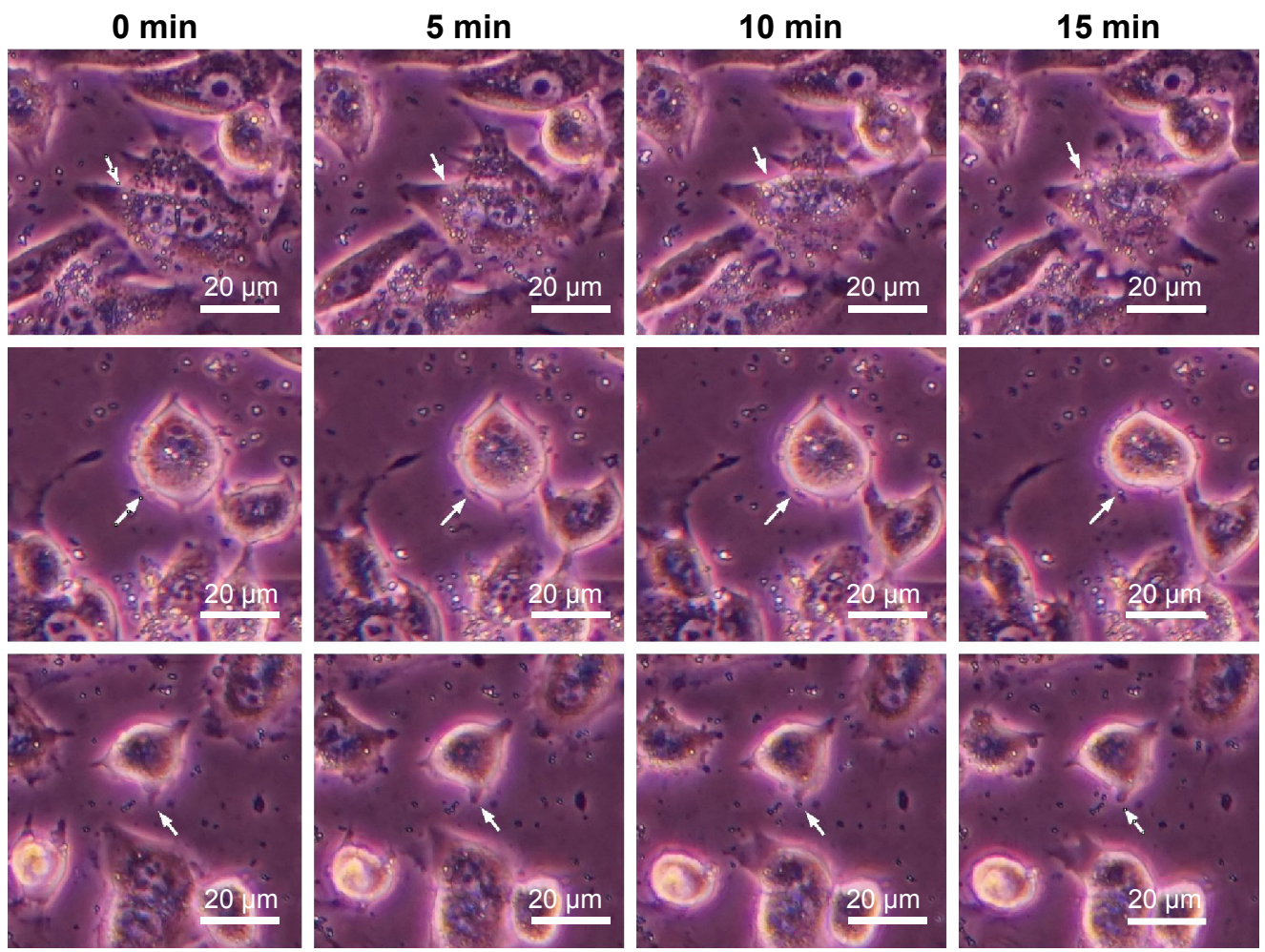

Figure S2 Series of images of microsphere-ingested 3T3 cells in a six-well culture plate after medium replenishment (5-min intervals).

Note: Arrows indicate cellular secretion of microspheres.

Abbreviation: min, minute.

International Journal of Nanomedicine

\section{Publish your work in this journal}

The International Journal of Nanomedicine is an international, peerreviewed journal focusing on the application of nanotechnology in diagnostics, therapeutics, and drug delivery systems throughou the biomedical field. This journal is indexed on PubMed Central, MedLine, CAS, SciSearch ${ }^{\circledR}$, Current Contents ${ }^{\circledR} /$ Clinical Medicine,

\section{Dovepress}

Journal Citation Reports/Science Edition, EMBase, Scopus and the Elsevier Bibliographic databases. The manuscript management system is completely online and includes a very quick and fair peer-review system, which is all easy to use. Visit http://www.dovepress.com/ testimonials.php to read real quotes from published authors.

\footnotetext{
Submit your manuscript here: http://www.dovepress.com/international-journal-of-nanomedicine-journal
} 